\title{
植物保护遗传学研究进展
}

\author{
李昂葛颂” \\ (中国科学院植物研究所系统与进化植物学开放研究实验室, 北京 100093 )
}

摘要: 保护遗传学是运用遗传学的原理和研究手段, 以生物多样性尤其是遗传多样性的研究和保护为核心的一门 新兴学科。近几十年来, 遗传学研究在生物多样性保护的理论和实践中发挥着越来越重要的作用。本文简要回顾 了保护遗传学的发展历史、研究方向和涉及的概念, 着重介绍了植物保护遗传学研究所取得的一些进展, 包括植物 系统发育重建和保护单元的确定、遗传多样性与物种和群体适应性之间的关系、群体遗传结构与保护策略的制定 以及植物遗传资源的鉴定和利用等方面的内容, 并强调保护遗传学研究是未来生物多样性和保护生物学研究中一 个亟待加强的研究领域。

关键词：保护遗传学，生物多样性，植物

中图分类号:Q16 文献标识码 : A 文章编号 : 1005-0094(2002)01-0061-11

\section{Advances in plant conservation genetics}

\section{Ang , GE Song}

Laboratory of Systematic and Evolutionary Botany, Institute of Botany , Chinese Academy of Sciences ,Beijing 100093

\begin{abstract}
Conservation genetics is a new field of research focusing on the studies and practices of biodiversity conservation based on the principles and techniques of population genetics. During the past decades, genetic studies have made increasingly great contributions to biodiversity conservation in theory and practice. In this paper, we briefly introduce the concept and history of conservation genetics, and highlight progress in plant conservation genetics. Four major aspects of conservation genetics in plants are addressed, including plant phylogenetic reconstruction and identification of conservation units, the relationship between genetic diversity and species fitness, population genetic structure and conservation strategies, as well as the identification and utilization of plant genetic resources. In addition, the great importance of genetic studies in plant conservation is discussed.
\end{abstract}

Key words : conservation genetics, biodiversity , plant

\section{1 前言}

生命在地球上出现至今,已经有三十多亿年的 历史。经过多次大绝灭和适应辐射, 各种生命形式 共同缔造了自然界的一片繁荣景象。然而, 近几百 年来, 由于世界人口的高速增长, 人类经济活动的不 断加剧,地球上的生物多样性正在急剧下降 (Frankham，1995)。尤其是在生物多样性十分丰 富的热带、亚热带发展中国家, 由于人口膨胀和经济 发展所带来的压力, 生态系统正受到严重的破坏, 大 量物种已经灭绝或处于濒危状态; 与此同时, 许多物
种的居群规模正在锐减, 种内遗传多样性急剧丧失 (葛颂，洪德元，1994; Meffe et al. ，1994； Avise \& Hamrick，1996)。与此同时,人类对生物资源的依 赖和需求却在日益增大。在这些严酷的事实面前， 人们终于认识到, 自然界中物种的多样性和进化潜 力是其自身生存和发展的基础, 保护物种及其所蕴 藏的遗传多样性就是保护生物多样性, 就是保护人 类赖以生存的资源, 就是保护人类自己(陈灵芝等， 1993 ; Meffe et al. , 1994 )。

20 世纪 70 年代,国际社会开始意识到人类经 
济活动的加剧正在对生态环境产生不利影响, 进而 对生物 (包括我们人类自身) 的生存带来直接的威 胁，从而把生物多样性的研究和保护提到了空前的 高度，一门新的综合性学科——保护生物学 (Conservation Biology) 也应运而生(蒋志刚等，1997 ;Meffe et al. , 1994)。随后, 有关的研究和保护活动也 在各国开展起来。在生物多样性三个主要层次, 即 遗传多样性、物种多样性和生态系统多样性中，遗传 多样性是指生物体内决定性状的遗传因子及其组合 的多样性，是物种多样性和生态系统多样性的基础 (葛颂，洪德元，1994;Avise \& Hamrick，1996)。任 何物种, 只有具备一定的遗传多样性才能抵御自然 界中的各种生存压力, 否则灭绝将是不可避免的 (Meffe et al. , 1994 ; Avise \& Hamrick ,1996)。

基于这一认识, 近 20 年来, 在保护生物学的研 究和实践过程中, 人们逐步将遗传学的原理和方法 运用于生物多样性的研究和保护, 成为保护生物学 中一个十分活跃的研究领域和方向 (葛颂, 洪德元, 1994 ; 湖志昂，张亚平，1997; 邹喻苹等，2001；Falk \& Holsinger , 1991 ; Avise \& Hamrick , 1996 ), 由此 而产生了一门新的学科一一保护遗传学 (Conservation Genetics)。本文试图就该学科的概念、发展历 史和研究内容, 尤其是在植物保护遗传学中取得的 一些成绩做一概述，以期推动国内该领域的研究。

\section{2 保护遗传学的概念、目的和研究内容}

尽管保护遗传学这一名词的提出和广泛应用的 时间并不很长, 但遗传学在资源和物种保护中的应 用则可以回溯到 20 世纪 30 年代。1937 年,Errington 和 Hamerstromsh 在野生动物管理方面首次提 出了“ Conservation Biology” 这一名词, 而 20 世纪二 三十年代，俄罗斯科学家 Vavilov 等通过对农作物 的研究已开始强调遗传多样性的重要性 ${ }^{\mathbb{1}}$ 。此后的 几十年间, 染色体、等位酶、DNA 等多种遗传分析手 段逐步得到广泛应用,为保护遗传学研究积累了大 量资料，所涉及的物种也从农作物扩展到野生动植 物和人类 (葛颂, 洪德元, 1994 ; 胡志昂, 张亚平, 1997 ; Meffe et al. , 1994)。尤其是 70 年代以后，分 子生物学和群体遗传学的迅猛发展, 为保护遗传学 研究的开展奠定了坚实的理论和技术基础。1978 年第一届国际保护生物学大会的召开,标志着该学 科的确立, 而在众多保护生物学研究者中, 有相当一
部分是从事遗传学和群体遗传学研究工作的。此 后, 保护遗传学方面的研究日益增多, 保护遗传学逐 步形成和完善了自己的理论体系和研究方法, 开始 以一门崭新学科的姿态出现 (Avise \& Hamrick, 1996 ; Holsinger，1996)。在此其间,由 Frankel 和 Soule 编著的《Conservation \& Evolution》 (1981) 和由 Schonewald-Cox 等编辑的《Genetics and Conservation》(1983) 对该领域的发展起到了积极的作用。 1991 年由 Falk 和 Holsinger 编辑的《Genetics and Conservation of Rare Plants》是对植物该领域研究的 一个较为全面的回顾和总结。如今, 保护遗传学已 经逐渐发展成为一门成熟的学科, 全世界许多学者 在从事这方面的研究, 有关的文献资料逐年增加 ( Meffe et al. , 1994 ; Loescheke et al. , 1994 ; Frankham , 1995 ; Avise \& Hamrick , 1996 ; Goldstein et al. , 2000)。2000 年,在国际著名的学术出版社 Kluwer Academic Publishers 的组织和推动下, 一份 名为 《Conservation Genetics》的国际性专业期刊在荷 兰创刊，标志着这门学科已逐步成熟并受到国际社 会的广泛关注。

根据 Woodruff( 1989,1990$)$ 的定义, 保护遗传学 就是运用遗传学的原理和研究手段, 以生物多样性 尤其是遗传多样性的研究和保护为核心的一门新兴 学科。实际上, 它可以说是一个多学科的综合, 融合 了群体遗传学、生态学、分子生物学、数学和系统与 进化等许多领域的研究内容, 兼有基础科学和应用 科学的性质。保护遗传学是在对濒危物种制定正确 的保护策略中应运而生的, 它首先要解决的问题是 确立保护单元、查明物种当前的遗传学状况 (即遗 传多样性水平、居群的分化程度等), 并据此决定对 其实施何种保护策略。由于保护遗传学是一门综合 性很强的学科, 其研究内容涉及物种保护的各个方 面。尽管不同学者有不同的观点 (Frankham, 1995； Avise \& Hamrick , 1996 ; Holsinger , 1996) ,但正如 Avise \& Hamrick (1996) 在《Conservation Genetics》书中所指出的, 其核心内容不外乎 5 个方面的内容, 即研究居群内遗传变异的水平及其意义; 居群内个 体间的亲缘关系式样; 居群的群体遗传结构和居群 间的进化关系, 种间界限和杂交; 以及物种或更高级

(1) 宿兵, 1996. 中国八种珍稀动物的遗传多样性与保护. 中国科学 院昆明动物研究所博士学位论文 
别类群间的系统发育关系。此外，物种的进化历史、 适应潜力和濒危的机制、交配系统及其进化机制、外 来种入侵的遗传后果、栽培群体经济形状衰退、法学 鉴定 (包括与贸易有关的品种和品系鉴定) 等等都 属于保护遗传学的研究范畴 (Milligan et al. , 1994 ; Frankham , 1995 ; Holsinger , 1996 ; Case et al. , 1998 ; Soule \& Hillis, 1998 ; Congiu et al. , 2000 ; Goldstein et al. ,2000)。

\section{3 植物保护遗传学所取得的成绩}

保护遗传学的原理和方法在野生动物中得到较 为广泛的应用, 如大熊猫 (Ailuropoda melanoleuca)、 白犀牛 (Ceratotherium simum)、红顶豚木鸟 (Picoides borealis) 和太平洋鲑鱼 (Oucorhynchus spp.) 等物种 的保护, 都是由于遗传学的介入而收益颇大 (胡志 昂 张亚平 ,1997; Meffe et al. , 1994 ; Avise \& Hamrick，1996; Soule \& Hillis，1998)。动物遗传资源的 研究、保护和利用也成为当前科学界讨论的焦点 (Morin 2000 ; Ryder et al. ，2000)。尽管植物的保 护遗传学起步相对较晚, 但近年来已取得了可喜的 进展。以下将简单介绍植物保护遗传学主要研究领 域的进展和取得的主要成绩。

\section{1 系统发育重建和物种保护单元的确定}

制定物种保护策略和措施的最重要一步是确定 需要优先保护的类群或物种。尽管迄今在科学上被 描述过的物种仅有 175 万种, 但估计地球上与人类 共栖共存的物种大约有 $1300 \sim 1400$ 万种 (Heywood et al. , 1995)。面对如此庞大的物种多样性和大量 物种处于灭绝边缘这一事实, 保护活动所能涉及的 物种只是其中很小的一部分, 并非所有类群对人类 都同等重要, 因此确定优先保护的类群是保护遗传 学要解决的重要问题之一。1986 年, Ryder 首次提 出了”进化显著单元 (evolutionarily significant units) (ESU) ”的概念，用来代表根据不同技术确定的、具 有明显适应性变异的一组群体 (Ryder，1986) ,由于 其在进化上的独特性而需要加以优先保护。ESU 的 概念已在动物的遗传管理和保护中得到广泛运用 ( 胡志昂 张亚平 , 1997; Crandall et al. , 2000)。

Moritz (1994) 曾对 ESU 给出了一个量化的定 义, 其基本含义是 :如果有 $\mathrm{A}$ 和 $\mathrm{B}$ 两个类群, 若它们 在线粒体 DNA 水平上互为单系群 (reciprocal monophyly）,并且在核基因座位上的基因频率已有显著
分化, 则认为 $\mathrm{A}$ 和 $\mathrm{B}$ 两个类群分属不同的 $\mathrm{ESU}$, 在 保护和遗传管理中应分别对待。实际上，根据遗传 上的独特性来确定保护类群主要是基于类群的分类 学地位和种间关系, 即利用分类学和系统发育方面 的资料来确定优先保护的类群 (胡志昂, 张亚平, 1997 ,邹喻苹等 2001)。例如，那些特有的单型属或 科应给予最优先的保护, 因为它们在分类或遗传上 最为独特因而不可替代 (Falk \& Holsinger，1991)。 在植物中, 对类群研究的不足和偏差常常导致对物 种濒危现状的错误判断 (洪德元等,1995)。因此, 利用遗传学资料和数据进行分类处理和澄清种间关 系在植物保护遗传学中就显得尤为重要。

茄科的一年生草本 Oryctes nevadensis 是Oryctes 属的唯一成员, 只出现在美国 Owens Valley 的 6 个 地点以及相邻的 Nevada 几个地点。1986 年统计只 发现 603 株个体, 是非常濒危的物种, 但未被列入濒 危物种名录。1991 年, Holsinger \& Gottlieb 提出, Oryctes nevadensis 完全应该加以优先保护, 不仅仅因 为该种只有 603 株个体, 而且因为其代表了一个独 特的进化类群。相反, 锦葵科 (Malvaceae) 的 Iliamna core i 被美国联邦政府列为濒危植物, 因为该种迄 今只发现有 1 个居群, 分布于美国 Virginia 州, 然而 该种的近缘种 I. remota 则分布于 Virginia、Indiana 和 Illinois 3 个州。Steward \& Excoffier (1996) 利用 RAPD 技术研究了这 2 个种的遗传多样性及其两者 之间的分类关系, 结果表明 2 个种间的遗传差异不 显著，因此提出把濒危种 I. corei 处理为 I. remota 的亚种，其保护的价值也相应下降。

沙参属 (Adenophora) 是桔梗科 (Campanulaceae) 内中等大小的属, 形态变异非常大, 因此种间关系模 糊, 许多种的分类地位存在争议,包括一些珍稀濒危 物种。葛颂等 (1995,1998) 和 Ge \& Hong (1994) 对 泡沙参复合体进行了物种生物学研究, 发现原有的 6 个种实质上只有 2 个种在分类学上成立, 其中一 个就是仅分布于四川金川、马尔康县境内的濒危物 种裂叶沙参 ( A. lobophyllya $)$ 。但是, 由于裂叶沙参 的标本非常少, 在分类学上是否成立曾有争议。对 这样一个濒危物种是否值得保护, 将在很大程度上 取决于它在分类上是否为一独立的物种, 即是否为 进化上的显著单元。为此, Ge et al. (1999b) 采用等 位酶技术对裂叶沙参及其近缘广布种泡沙参 $(A$. potaninii) 进行了遗传多样性和种间关系的研究, 通 
过对 5 个裂叶沙参居群和 7 个泡沙参居群等位酶数 据的分析表明, 这 2 个种内居群间的遗传分化都很 小,而种间遗传分化非常明显（图 1 ）,因此认为裂叶 沙参是一个独立的物种, 值得加以保护。

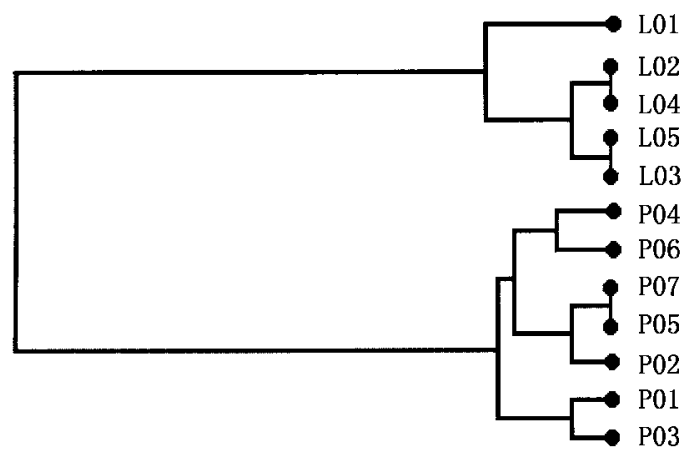

$\begin{array}{llllllll}0.14 & 0.12 & 0.10 & 0.08 & 0.06 & 0.04 & 0.02 & 0.00\end{array}$ 遗传距离 Genetic distance

图 1 裂叶沙参 (Adenophora lobophylla) 5 个群体及其近缘 广布种泡沙参 (A. potaninii) 7 个群体之间的遗传关系。 $\mathrm{L}$ 代表裂叶沙参;P 代表泡沙参 (引自 Ge et al.,1999b)

Fig. 1 Phenogram of genetic distance for five populations of $A$. lobophylla and seven populations of A. potaninii, based on Nei's (1978) unbiased genetic distance coefficient. L represents $A$. lobophylla and $\mathrm{P}$ represents A. potaninii.

与此十分相似的一个例子来自对兰科杓兰属 Cypripedium kentuckiense 的研究。该种是一个稀有 物种,主要分布在美国 Arkansas 州,但关于这个种 成立与否曾经有过争议。有人认为它只是广布种 C. parviflorum var. pubescens 的一个极端形式,但等 位酶数据支持将其作为一个独立的种, 因而有必要 对其采取保护措施（Case et al. ，1998)。杂交是植 物界广泛存在的一种现象, 故杂种鉴定对保护单元 确定以及保护策略和措施的制定具有重要意义。 Crawford et al. (1993) 用等位酶和 RAPD 检测了智 利附近 Masatierra 岛上的一种珍稀植物 Margyracaena skottsbergii 的杂交起源问题。这个种曾被认为是 该岛上特有种 Margyricarpus digynus 与一种入侵植 物 Acaena argentea 杂交产生的，而原产该岛的 Acae$n a$ ovalifolia 也曾被认为是亲本之一。但 Crawford $e t$ al. 的研究表明, A. ovalifolia 不是 M. skottsbergii 的 亲本。由于杂交种不可育,自然状态下只能与 $A$. argentea 同时存在,故保护 M. skottsbergii 的同时必 须保护其种质来源 A. argentea。Smith et al. (1996) 采用 RAPD 标记对夏威夷苦苣苔科 Cyrtan$d r a$ 属的研究表明,该属有 17 个物种均为杂种起源 (邹喻苹等 2001)。类似采用遗传标记方法确定物
种保护单元的例子还很多 (Castiglione et al. , 1993 ; Rossetto et al. ,1999 ; Goldstein et al. ,2000 ; Szalanski et al. ,2001)。

\section{2 遗传多样性与物种和群体的适应性}

Meffe et al. (1994) 在其编著的《Princeples of Conservtion Biology 》一书中，从 3 个方面概括了遗传 多样性的重要意义。首先, 根据自然选择的基本原 理, 居群的进化速率与其遗传多样性成正比, 换句话 说,一个群体中遗传多样性的下降意味着其适应环 境变化能力的下降;其次, 群体遗传学研究表明, 群 体中的杂合性也即居群内遗传变异的水平, 是与其 适合度成正相关的; 第三, 全球的遗传多样性中蕴藏 了所有生命过程的信息，任何遗传多样性的丢失都 会导致有机体对环境变化的适应能力降低,同时也 使人类丧失了具有潜在应用价值的生物信息和资 源。

对许多动物物种的研究表明, 杂合性水平与其 适合度有显著的正相关 (Meffe et al. , 1994) (图 2)。 近年来的研究表明, 遗传多样性的丧失将对物种生 存带来直接的不利影响 (Saccheri et al. , 1998 ; Soule \& Mills，1998)。植物中的类似报道也有不 少。Mitton \& Grant (1980) 在对颤杨 (Populus tremuloide) 的研究中发现,在所分析的年龄、杂合度、性 别、海拔等因素中, 有 2 个因素与平均生长率之间存 在显著的正相关，有 1 个因素存在显著负相关(表 1 ) 其中个体的杂合度与平均生长率呈显著正相 关。与此同时, 他们还发现在西黄松 (Pinus pondero$s a)$ 的群体中, 幼苗的 $F$ 值比种子要低, 说明杂合体 较纯合体有生存优势 (Mitton \& Grant，1984)。最 近，对百合科濒危物种木根麦冬 (Ophiopogon xylorrhizus) 的研究表明, 结实率和家系的远交率及母本 的杂合度显著相关 (图 3, He et al. 1998)。而且不 同世代间居群遗传变异的研究表明，子代群体在生 长发育至成年群体时，居群中基因杂合度显著增高， 说明在这一过程中, 自然选择淘汰了大量的纯合度 高的个体, 证实了杂合度高的个体具有较高的适合 度 ( He et al. , 2001)。对裸子植物的许多研究发 现, 自交或近交会明显降低群体中的杂合度, 从而导 致这类异交植物产生明显的近交衰退( Savolainen， 1994 ; Ge et al. , 1998 )。Antonovics \& Ellstrand (1984) 对 Anthoxanthum odoratum 的研究表明,在该 物种的群体中, 频率低的基因型适合度高 (即符合 

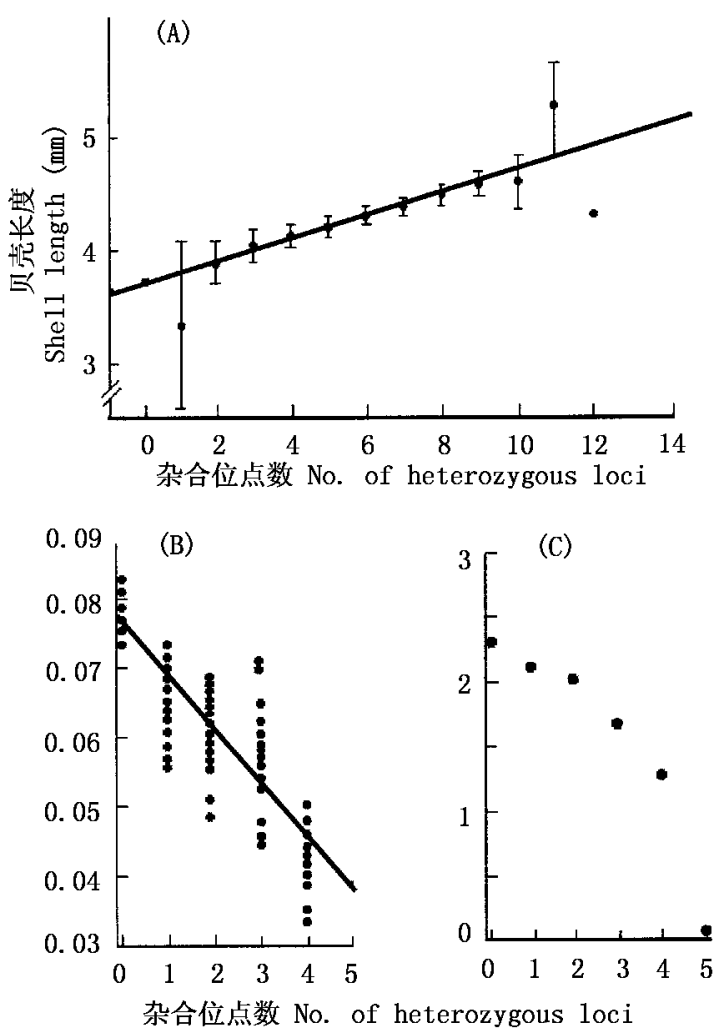

图 2 杂合性水平和 3 个与适合度有关的性状之间的关系 A. 一种蛤类 (Mulinia lateralis) 个体的生长速率;B. 美洲牡蚛 (Crassostrea virginica) 的耗氧量，耗氧量低说明代谢效率高; C. 彩虹鲑鱼 (Oncorhynchus mykiss) 形态上的不对称性, 不对 称性高说明发育上的不稳定 (引自 Meffe et al. , 1994)。

Fig. 2 Three examples of correlations between heterozygosity level and three fitness-related characters. A. Growth rate in the coot clam Mulinia lateralis over a two-week period after collection as larvae; B. Oxygen consumption in the American oyster (Crassostrea virginica), low consumption indicates greater metabolic deficiency; C. Morphological asymmetry in rainbow trout (Oncorhynchus mykiss) ; greater asymmetry indicates less developmental stability.

表 1 颤杨 (Populus tremuloide) 平均生长率的多元回归 Table 1 Multiple regression of average growth rates of Populus tremuloide

\begin{tabular}{lcc}
\hline & $\begin{array}{c}\text { 系 数 } \\
\text { Index }\end{array}$ & $\begin{array}{c}\text { 显著性 } \\
\text { Significance }\end{array}$ \\
\hline 多元回归 Multiple regression & 0.606 & $P<0.001$ \\
各自变量 Independent variables & & \\
年 龄 Age & -0.152 & $P<0.001$ \\
杂合度 Heterozygosity & 1.637 & $P<0.05$ \\
性 别 Sex & 1.425 & $P>0.20$ \\
海 拔 Elevation & -0.017 & $P<0.01$ \\
\hline
\end{tabular}

依频选择假说），与亲本在遗传上有差异的后代具 有生存优势, 证明遗传多样性高有利于物种的生存。 但另一方面,有些研究实例表明,遗传变异性与
群体或个体的生存之间不存在相关关系。Holsinger (1996) 举出智利 Channel 岛狐狸的例子,说明低水 平的遗传多样性并不一定影响物种的生存能力, 他 同时认为分子标记所显现出的多样性水平并不能可 靠地反映与选择压有关的性状的变异程度。对欧洲 赤松 (Pinus sylvestris) 的研究也没有发现杂合度与群 体的适合度有明显的正相关 (Savolainen \& Hedrick， 1995 )。事实上, 关于遗传多样性与物种和群体的 适应性之间的关系一直存在着不同的观点和争论 (张大勇,姜新华,1999;Lande，1988；Holsinger， $1996)$ 。正如许多学者所强调的, 在很多情况下, 遗 传多样性的丧失更可能是物种濒危的症状而不是濒 危的原因（张大勇，姜新华，1999; Meffe et al. ， 1994 ; Holsinger，1996)。因此，有关杂合度与物种 和群体适应性之间的关系及其机制还需要进一步深 入研究。
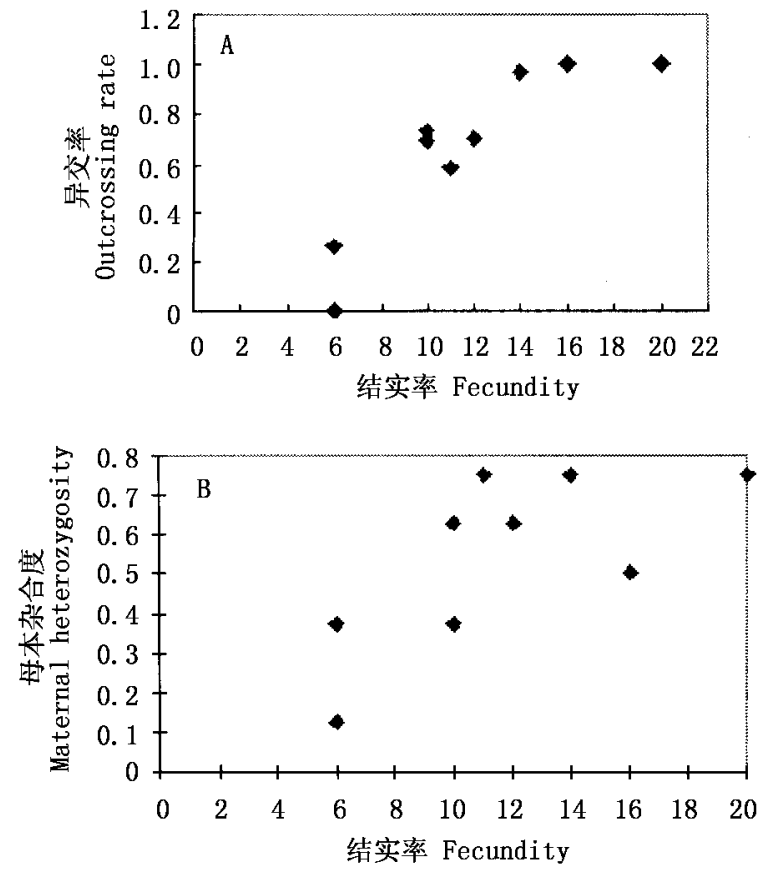

图 3 木根麦冬 (Ophiopogon xylorrhizus) 结实率与家系远 交率及母本杂合度之间的相关关系。

A. 结实率与家系多位点远交率之间的相关关系。B. 结实 率与母本杂合度之间的相关关系。(引自 He et al. ,1998)

Fig. 3 Relationships between fecundity and multilocus outcrossing rates of family and maternal heterozygosity. A. Relationships between fecundity and multilocus outcrossing rates of families ; B. Relationship between fecundity and maternal heterozygosity.

\section{3 群体遗传结构与保护策略的制定}

植物群体的遗传变异水平和群体遗传结构是其 进化历史、分布范围、生活型、繁育方式、种子散布机 
制等各种不同因素综合作用的结果，与其适应性和 进化潜力密切相关, 故检测植物的遗传变异水平及 其空间结构，是探讨上述各种进化因素的前提，同时 关系到物种保护和复壮的策略和措施的制定( 葛 颂，洪德元，1994; 葛颂，1997 ;Meffe et al. ，1994)。 尤其是针对稀有和濒危的物种以及受人类直接干扰 和破坏的物种，在缺乏基本的遗传学信息的情况下 很难制定科学合理的保护和抢救措施( 邹喻苹等, 2001 ; Falk \& Holsinger 1991)。

目前，人们已利用等位酶以及各种 DNA 分子标 记对大量物种进行了研究，取得了可喜的成绩，国内 外有关的实例很多 (葛颂，1997; 胡志昂，张亚平， 1997 ; 邹喻苹等，2001；Hamrick \& Godt，1990; Falk \& Holsinger，1991)。例如，在对我国特有濒危 植物银杉 (Cathaya argyrophylla) 的研究中，根据 12 个酶系统 25 个等位酶位点对 8 个居群共 101 个个 体的检测，发现该物种居群水平上的遗传多样性只 相当于其他裸子植物的 $1 / 2$ 到 $1 / 3$, 然而其居群间 的遗传分化则十分强烈，基因分化系数(Gst) 高达 0.441 , 是其他裸子植物平均值的 6 倍 ( Ge et al. ， 1998 )。采用 RAPD 技术对该种进行的分析也得到 了相同的结果 (汪小全等,1996)。因此，结合化石 资料推测，银杉在其进化历史上经历了严重的瓶颈 效应, 小居群的相互隔离和随之而来的遗传漂变是 导致其遗传变异大幅下降、居群间遗传分化加大的 主要原因 (葛颂等, 1997 ; Ge et al. , 1998)。

在众多影响物种群体遗传结构的因素中, 繁育 系统起着十分关键的作用 (葛颂，1997; Hamrick \& Godt ，1990)。对火烧兰属 (Epipactis) 群体遗传结构 与繁育系统的研究是个典型的例子。Ehlers \& Pedersen (2000) 采用等位酶技术研究了丹麦境内的 3 个火烧兰属物种的遗传多样性和地理分布，结果发 现物种的繁育系统与群体的遗传结构密切相关。异 交广布的 E. helleborine ssp. helleborine 显示出高水 平的遗传变异 $(P=73.6, H e=0.274)$,而且绝大部 分变异 $(91 \%)$ 存在于群体之内, 而专性自交种 $E$. phyllanthes 在检测的全部位点上均为单态 (Ehlers \& Pedersen , 2000)。Scacchi et al. (1991) 对 3 种繁育 方式不同的头荢兰 (Cephalanthera spp.) 的研究也得 出了相似的结论。在对我国云南热带雨林的濒危物 种木根麦冬的研究中，Ge et al. (1997) 发现, 尽管该 种仅分布于 $30 \mathrm{~km}^{2}$ 范围内, 但群体的分化和隔离十
分强烈,他们据此推断该种可能发生了较为严重的 近交或自交(Ge et al. ，1997)。后来的进一步研究 证实了这一推断，该种出现了交配系统的分化及较 为严重的自交衰退, 这可能是其濒危的重要原因之 一( He et al. , 1998)。

在对物种实施保护计划过程中，原位保护地点 的选择和迁地保护的采用策略都需要遗传学资料作 为基础 (Falk \& Holsinger , 1991; Hogbin \& Peakall, 1999 )。对一个遗传变异主要存在于居群之内的物 种 (如大多数风媒异花授粉的裸子植物) 和一个遗 传变异主要分布于居群之间的物种 (如许多自花授 粉的一年生草本植物) 应具有完全不同的取样和保 护方针(邹喻苹等,2001)。尽管稀有和濒危物种遗 传变异的平均水平要明显低于广布种，但不同物种 的多样性水平和居群遗传结构会有很大的不同 (葛 颂,洪德元，1999;Hamrick \& Godt，1990)。因此， 对一个物种应采取什么样的保护策略和措施, 必须 建立在对该物种群体遗传学研究的前提下。如前述 的银杉就具有与其他裸子植物明显不同的群体遗传 结构，从而应采用不同的取用和保护策略。在欧洲 阿尔卑斯山 (Alps) 分布的虎耳草科 Saxifraga cernua 具有十分独特的遗传结构。Bauert et al. (1998) 采 用 RAPD 技术对阿尔卑斯山 3 个地区共 7 个居群的 检测发现, 在同一地区, 居群内和居群间均没有发现 RAPD 谱带的差异, 尽管每个居群均检测了 30 个左 右的个体，但地区之间差异却很明显 (Bauert et al. , 1998 )。换句话说, 该物种的遗传变异全部分布于 群体之间, 故此类物种的保护应把重点放在群体间 而不是群体内。对我国保护植物硬叶兒兰 (Paphiopedilum micranthum) 的研究表明, 由于人为采摘和 生境片断化等因素,该物种的遗传多样性已明显低 于其他兰科植物, 而群体间的遗传分化则明显偏高。 在所检测的 4 个群体中, 有 2 个群体的遗传多样性 显著高于其他群体，因此在原位保护中更有价值。 但如要进行迁地保护则应对尽可能多的群体进行 ( Li et al. 2002)。

对澳大利亚芸香科 Zieria prostrata 的研究是植 物保护遗传学研究中比较成功的例子。该种仅分布 于沿海岸 $3 \mathrm{~km}$ 范围内 ,Hogbin \& Peakall (1999) 采 用 RAPD 分析技术发现该种遗传变异在居群间所 占比例很高 $(37 \%$ ) 结合其他遗传学研究结果对保 护行动提出了 4 点意见 : 1) 任何一个居群的丢失都 
会对遗传变异造成很大损失 2 ) 迁地保护必须考虑 分布范围内的所有居群 3 ) 如果要进一步实施迁移 计划，应考虑进行居群间混合繁殖;4) 对一个曾被 认为是取自现已灭绝居群的个体的分析发现，这个 个体在遗传上与现存居群个体十分相近，从而对该 个体的来源提出了疑问,并放弃了将其迁移到原地 的保护计划 (Hogbin \& Peakall, 1999)。

物种不同的生活史特征对保护措施的制定也有 重要的影响。例如, 克隆现象是植物中常见的一种 繁殖策略，克隆生长往往会使群体的实际规模难以 估计，从而错误地估计遗传上的个体数目; 克隆习性 的强弱和克隆的空间结构对取样策略的制定也有直 接的影响 (葛颂等，1999;Sydes \& Peakall，1998; Xie et al. ,2001)。He et al. (2000) 在对木根麦冬 的研究中指出, 该物种的基因型在群体内呈现明显 的空间结构，因此在保护过程中必须注意取样对所 有基因型的代表性。Swensen et al. (1995) 采用等位 酶、RAPD、和 RFLP 等多种手段，对美国 California 海岸附近 Santa Cruz 岛上的濒危植物 Malacothamnus fasciculatus var. nesioticus 进行了检测, 发现在现存 的 2 个群体中只有 5 个基因型, 为进一步采取人工 建群等措施提供了科学依据。

对于稀有和濒危物种来说，在探讨其进化的历 史和揭示其濒危机制的前提下，更重要的是应该提 出能使该物种及其群体得以恢复和复壮的可行的途 径和方法。前述我国的一级保护植物银杉是十分濒 危的树种，其生长缓慢、结实量小、种子发芽率低，因 而天然更新困难。Ge et al. (1998) 根据其群体间遗 传分化极高这一独特的群体结构，提出选取遗传差 异明显的群体进行群体间人工控制杂交,是促使该 物种复壮的可行方法之一。由于生境的大规模破 坏，澳大利亚西部山龙眼科 (Proteaceae) 的多年生木 本 Grevillea scapigera 已处于灭绝的边缘，只剩下 4 个居群，个体总数只有 27 株, 是亟待抢救的物种。 为此, Rossetto et al. (1995) 采用 RAPD 标记对该种 现存居群的遗传多样性进行了研究, 同时对已开展 的移植策略进行了评估。结果发现居群间几乎没有 遗传差异, 说明相互移植不会有不利的遗传效应 ( Rossetto et al. ，1995)。Wong \& Sun (1999) 通过 人工杂交、等位酶和 RAPD 研究了香港濒危的兰花 Goodyera procera 的生殖生物学和群体遗传结构, 并 在此基础上提出了相应的保护措施。

\section{4 植物遗传资源的鉴定、保护和利用}

遗传资源就是蕴藏在各种生物中的遗传变异, 包括野生生物 (动植物、微生物) 中存在的遗传变 异。20 世纪后半叶, 随着遗传资源侵蚀程度的不断 加剧，植物资源采集和保存的观念已发生了很大的 变化。从强调采集具有直接利用价值的特定基因、 居群或生态型资源到重视采集对现在和将来都具有 保护和利用价值的遗传资源 (Tanksley \& McCouch, 1997 )。由于通常很难确定哪一类基因更有价值从 而在采样中加以优先, 所以遗传资源的保护应使每 个基因位点上尽可能多的变异得以保存, 因为任何 一种野生生物都是可利用的潜在的遗传资源，不知 哪一天就会被科学家派上用场(邹喻苹等 2001)。

随着生物多样性保护日益受到重视, 大规模的 资源调查和收集工作也在全球范围内开展起来。 Morin ( 2000 ) 在刚刚创刊的国际期刊《Conservation Genetics》第 1 卷第 1 期上撰文,将如何有效地保护 遗传资源看成是新世纪的机会和挑战, 并认为开展 保护遗传学研究已迫在眉睫, 其紧迫性决不亚于对 遗传资源的收集和保护。以目前世界上最重要的 7 种农作物为例, 迄今在世界主要种质中心保存的资 源就数以百万计 (表 2)。面对这样庞大的种质资 源，如何进行有效的管理和利用是摆在人们面前的 首要问题。为此,遗传学的原理和方法为快速有效 地进行资源鉴定、评估和利用提供了基本的手段 ( 贾继增, 1996; ;邹喻苹等, 2001; Loescheke et al. , 1994 ; Tanksley \& McCouch , 1997 ; Gilbert et al. , 1999 )。

表 2 一些重要作物在世界主要种子库中保存的样品条目 数量( 据 Tanksley \& McCouch , 1997)

Table 2 Estimated number of seed bank entries worldwide for selected crops (Tanksley \& McCouch , 1997)

\begin{tabular}{lcc}
\hline 作物 & 条目 & $\begin{array}{c}\text { 野生种所占\% } \\
\text { Crops }\end{array}$ \\
Entry & 410000 & Percentage of wild species \\
\hline 小麦 Wheat & 215000 & 60 \\
水稻 Rice & 100000 & 10 \\
玉米 Maize & 100000 & 30 \\
大豆 Soybean & 42000 & 40 \\
土豆 Potato & 32000 & 70 \\
西红柿 Tomato & 30000 & 20 \\
棉花 Cotton & &
\end{tabular}

以世界头号粮食作物水稻为例，仅中国的地方 品种就有近万个 ( 陈灵芝等, 1993 ) ,国际水稻研究 所（IRRI）收集的稻属资源则多达 80000 多份，构成 了一个庞大的稻种基因源, 加上水稻所在的稻属还 
有 20 多个野生物种, 共同构成了水稻遗传改良的宝 贵资源 (卢宝荣,1998 ;Virk et al. , 1995)。然而,世 界主要种子库所保存的水稻样品中, 野生种只占 $10 \%$ (表 2)。近些年来, 各种遗传标记在对水稻及 其野生资源的研究、鉴定和利用方面起到了关键性 的作用。Martin et al. (1997) 尝试采用 RAPD 对 IRRI 种质库中的 93 份材料进行了鉴定, 取得了很好 的效果。在所研究的 93 份材料中，他们发现有 9 份 样品鉴定错误。同样,Virk et al. (1995) 用 RAPD 技 术证实原来所怀疑的 3 对样品中有 2 对是重复样 品。在包含 24 个物种的稻属中, 由于分类中可依据 的形态性状少, 且许多形态性状在物种内变异较大， 加上杂交事件和多倍体的普遍存在，使得稻属一些 物种的鉴定很困难, 具有不同染色体组的物种也往 往会出现鉴定错误(卢宝荣等,2001)。最近,Ge et al. (2001) 提出了一种快速而可靠地鉴定稻属染色 体组的方法，通过 PCR 扩增的 Adhl 和 Adh2 两个基 因片段的 RFLP 分析, 可以快速地鉴别出稻属全部 10 个染色体组，从而为稻属染色体组和物种的鉴定 提供了一种新的方法。类似在物种和变种以及品种 水平上的鉴定工作还有很多（邹喻苹等，2001；Cas-

tiglione et al. ,1993; Martin et al. ,1997 ; Congiu et al. 2000)。

栽培植物的祖型或野生近缘种中存在着十分丰 富的遗传多样性。目前在生产实践中普遍存在人工 繁殖群体的经济性状衰退, 这与其遗传多样性的降 低有密切关系。缺乏对作物近缘野生种的搜集、研 究和利用已严重地阻碍了当前作物遗传改良和育种 工作的进一步发展。因此, 研究和利用栽培植物野 生近缘种中的遗传变异, 是对其进行遗传改良的关 键 (贾继增，1996; Tanksley \& McCouch，1997)。 Ge et al. (1999a) 利用 RAPD 技术对采自中国和巴 西的普通野生稻 (Oryza rufipogon) 的天然居群进行 了对比研究, 希望了解这两地天然居群中遗传变异 水平和居群遗传结构的异同,同时探讨巴西普通野 生稻的分类地位。结果发现, 野生稻居群中含有丰 富的遗传变异。中国和巴西居群的对比分析表明, 来自中国普通野生稻的变异性要明显高于来自巴西 的居群, 中国 4 个居群的多态片断百分比为 $30.5 \%$ $\sim 43.2 \%$ (平均 $55.8 \%$ ), 而来自巴西 4 个居群的 多态片断百分比只有 $6.3 \% \sim 27.4 \%$ (平均 $41.1 \%$ )。居群遗传结构的分析表明, 总的遗传变
异中有 $61.8 \%$ 存在于中国和巴西 2 个地区之间, 每 个居群内个体间的变异性占 $23.3 \%$, 而地区内居群 间的变异性只分别占 $14.9 \%$ 。这些结果对进一步 最大限度地收集该种的遗传资源以及针对适应性性 状设计采样策略具有指导作用 (Ge et al. , 1999a)。 迄今, 通过不同的分子标记技术对中国全部 3 种野 生稻的群体遗传结构进行了初步的研究, 为这些重 要资源的保护和利用奠定了良好的基础 (Ge et al. , 1999a ; Gao et al. ,2000 ; Gao et al. ,2001 ; Qian et al. ,2001; Xie et al. ,2001)。

Szalanski et al. (2001) 用 PCR-RFLP 方法对美 国一种稀有绶草 (Spiranthes diluvialis) 进行研究发 现, 其群体间和群体内均不存在遗传变异, 其遗传多 样性的缺乏为进一步制定长期的保护策略提供了重 要的信息。Rio et al. ( 1997 ) 对 2 个土豆野生种的研 究发现, 种质库中保存的 1958 年和 1978 年采集的 样品与 1992 年再次采集的群体样品相比, 在遗传组 成上的分化已十分明显, 出现了种质库中所没有的 一些新的变异, 说明原位保护对遗传资源的保存具 有重要价值。

在正确收集和保存种质的基础上,高效利用这 些种质资源是人们追求的目标。为此, Marshall (1990) 提出了“核心种质” 的概念。它是指只占全 部种质 $10 \%$ 左右、但能最大限度的代表种质资源遗 传多样性的那些样品, 也就是遗传改良中应最先被 利用的种质资源。Schoen \& Brown (1993) 利用计算 机模拟的方法, 研究了建立核心种质几种主要方法 的效率, 即固定取样法 (每个地区取一定数目的样 本构成核心种质, C 策略), 比例取样法 (按每个地 区所具有的样本数成比例取出样本构成核心种质, $\mathrm{P}$ 策略), 对数取样法( 按每个地区所具有的样本数 按对数比例取出样本构成核心种质, L 策略), 随机 取样法 (随机地从每个地区取出样本构成核心种 质, R 策略) 和标记辅助取样法 (根据分子标记所体 现的多样性进行取样,M策略) ( Schoen \& Brown, 1993 )。结果发现, 若以获得最丰富的等位基因为 标准, 根据分子标记的辅助取样法是最为有效的方 法 (图 4)。

对其他农作物及其近缘野生种遗传资源研究的 实例还有很多(贾继増，1996; 胡志昂, 张亚平, 1997 ;卢宝荣, 1998 ; 黄宏文等, 2000 ; 邹喻苹等, 2001 ; Loescheke et al. ,1994 ; Gilbert et al. ,1999)。 


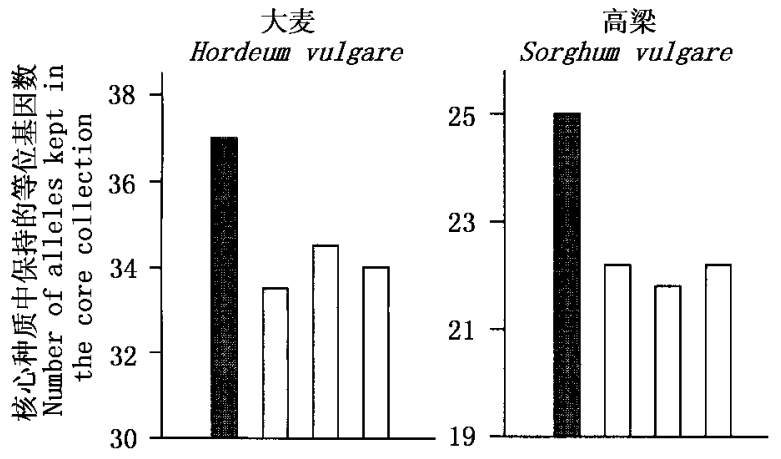

图 4 核心种质篮选过程中不同方法的效率

根据 Schoen \& Brown,1993 绘制。实心柱代表分子标记方 法 ;空心柱代表其他方法

Fig. 4 Efficiencies of different methods of core collection. Solid columns represent molecular makers. Hollow ones represent other methods ( Schoen \& Brown , 1993 )

\section{4 结束语}

作为保护生物学中一个较新的领域，保护遗传 学研究只能说是刚刚起步,随着保护生物学、遗传学 以及相关学科的发展，保护遗传学也在不断地完善 和提高。尽管遗传学原理和技术在区分保护单元、 了解遗传变异的水平和分布状况、推测进化历史和 指导保护措施的制定发挥着重要作用，但遗传学数 据仅仅为保护策略的制定提供了一个出发点和基础 性的资料，生物多样性和物种保护还将涉及方方面 面的许多因素 (Avise,1996)。如果对生物赖以生存 的生态系统以及物质能量流动缺乏了解, 仅仅研究 遗传多样性本身其价值是很有限的（张大勇，姜新 华, 1999 ; Lande , 1988 ; Meffe et al. , 1994 ; Holsinger，1996)。从实际应用的角度上看, 保护遗传学 也存在一定的局限性。遗传学研究费用较高, 相对 不易掌握, 在很多情况下还会被误用; 而且, 遗传学 研究必须建立在获得合适的动植物组织材料并妥善 处理的前提下等 (Meffe et al. ，1994; Holsinger， 1996 )。另一方面, 不同的遗传标记和方法所得出 的结果会有差别,如何在物种保护中有效和准确地 分析和利用遗传数据也是有待完善的问题( 李昂 等, 2000 ; 钱韦, 葛颂, 2001; Wolfe \& Liston， 1998 )。

尽管如此, 保护遗传学研究仍然是亟待加强的 一个研究领域, 尤其是对于我国这样一个物种资源 丰富而生物多样性又面临严重威胁的国家, 加快保 护遗传学研究十分必要。由于技术和经费等方面的 限制, 加上人们往往看重研究的实用性, 迄今在选择
研究对象时往往格外关注那些与人类密切相关的类 群, 如栽培作物的野生近缘种以及某些具有重要经 济或社会效益的野生动物( 宿兵, 1994; 胡志昂, 张 亚平,1997;卢宝荣，1998; 黄宏文等,2000;邹喻苹 等 2001 ; Meffe et al. , 1994)。大量野生动植物仍得 不到足够的重视, 更缺乏基本的研究。例如, 在兰科 (Orchidaceae) 这一被子植物第二大科中，绝大多数 物种以其䎦丽或芳香的花朵闻名于世, 具有很高的 观赏价值, 因此在巨大经济利益的驱动下, 很多野生 兰花种类从栖息地转移到了花卉经营者和爱好者手 中。更为严重的是, 分布着大量兰花种类的热带、亚 热带地区, 大面积森林遭到破坏, 兰花生存的生境日 益萎缩。这种全球范围内的生境退化和丧失加上人 为破坏是导致是大量兰花变为稀有、濒危以致灭绝 的主要原因 (IUCN/SSC Orchid Specialist Group, 1996 )。当人们已经意识到问题的严重性并着手制 定各种政策和法规时,却发现我们对这些物种的了 解和认识仍相当贫乏。迄今可检索到的有关兰花保 护遗传学研究的文献与该类群的多样性和重要性远 不相称, 大多数保护活动都是在缺乏基本的生物学 研究的情况下进行的 (IUCN/SSC Orchid Specialist Group , 1996)。对具有重要经济价值的兰花尚且如 此, 对其他动植物的研究则更为欠缺。Falk \& Holsinger (1991) 曾指出 我们不可能保护好我们所不了 解的”。因此, 全面开展保护生物学研究, 包括保护 遗传学研究是解决生物多样性危机、挽救成千上万 物种的根本出路, 也是合理的资源开发和利用的前 提。

\section{参考文献}

陈灵芝 (主编), 1993. 中国的生物多样性一一现状及其保护 对策. 北京: 科学出版社, $31 \sim 113$

葛颂, 1997. 植物群体遗传结构研究的回顾和展望. 见: 李承 森 (主编), 植物科学进展 (第 I 卷). 北京: 高等教育出版 社, $1 \sim 15$

葛颂, 洪德元, 1994. 遗传多样性及其检测方法. 见: 钱迎 倩, 马克平 (主编), 生物多样性研究的原理与方法. 北 京: 中国科学技术出版社, $123 \sim 140$

葛颂, 洪德元, 1995. 泡沙参复合体 (桔梗科) 的物种生物学 研究 III. 性状的遗传变异及其分类价值. 植物分类学 报, 33(5): $433 \sim 443$

葛颂, 洪德元, 1998. 泡沙参复合体 (桔梗科) 的物种生物学 研究 IV. 等位酶水平的变异和分化. 植物分类学报, 36 (6) : $581 \sim 589$

葛颂, 洪德元, 1999. 濒危植物裂叶沙参及其广布种泡沙参 遗传多样性的对比研究. 遗传学报, 26: $410 \sim 417$

葛颂, 王海群, 张灿明, 洪德元, 1997. 八面山银杉林的遗传 
多样性和群体分化. 植物学报, 39(3)：266 271

葛颂, 王可青, 董鸣, 1999. 毛乌素沙地根茎灌木羊柴的遗传 多样性和克隆结构. 植物学报, 41(3)：301 306

洪德元, 葛颂, 张大明, 汪小全, 程树志, 1995。植物濒危机制 研究的原理和方法. 见: 钱迎倩, 甄仁德 (主编), 生物多 样性研究进展. 北京: 中国科学技术出版社, 125 133

胡志昂, 张亚平 (主编), 1997. 中国动植物的遗传多样性. 杭州: 浙江科学技术出版社

黄宏文, 龚俊杰, 王圣梅, 何子灿, 张忠慧, 李建强, 2000 . 猕猴桃属 (Actinidia) 植物的遗传多样性. 生物多样性, 8: $1 \sim 12$

贾继增, 1996. 分子标记种质资源鉴定和分子标记育种. 中 国农业科学, 29(4): 1 10

蒋志刚, 马克平, 韩兴国 (主编), 1997. 保护生物学. 杭州: 浙 江科学技术出版社

李昂, 王可青, 葛颂, 2000. 不同采样策略对细距堇菜遗传多 样性估算的影响. 植物学报, 42(10): 1069 1074

卢宝荣, 1998. 稻种遗传资源多样性的开发利用和保护. 生 物多样性, $6: 63 \sim 72$

卢宝荣, 葛颂, 桑涛, 陈家宽, 洪德元, 2001。稻属分类的现状 及存在问题. 植物分类学报, 39: $373 \sim 388$

钱韦, 葛颂, 2001. 居群遗传结构研究中显性标记数据分析 方法初探. 遗传学报, 28(3):244 255

汪小全, 邹喻苹, 张大明, 洪德元, 1996. 银杉遗传多样性的 RAPD 分析. 中国科学 (C 辑), 26(5):436 441

张大勇, 姜新华, 1999. 遗传多样性与濒危植物保护生物学 研究进展. 生物多样性, 7:31 37

邹喻苹, 葛颂, 王晓东, 2001。系统与进化植物学中的分子标 记. 北京: 科学出版社, $140 \sim 149$

Avise J C and J L Hamrick, 1996. Conservation Genetics, Case Histories from Nature. Chapman \& Hall, New York

Bauert M R, M Kalin, M Baltisberger and P J Edwards, 1998. No genetic variation detected within isolated relict populations of Saxifraga cernua in the Alps using RAPD markers. Molecular Ecology, 7: $1519 \sim 1527$

Case M A, H T Mlodozeniec, L E Wallace and T W Weldy, 1998. Conservation genetics and taxonomic status of the rare Kentucky Lady's slipper: Cypripedium kentuckiense (Orchidaceae) . American Journal of Botany, 85: 1779 1786

Castiglione S, G Wang, G Damiani, C Bandi, S Bisoffi and F Sala, 1993. RAPD fingerprints for identification and for taxonomic studies of elite poplar (Populus spp.) clones. Theoretical and Applied Genetics, 87: $54 \sim 59$

Congiu L, M Chicca, R Cella, R Rossi and G Bernacchia, 2000. The use of random amplified polymorphic DNA ( RAPD) markers to identify strawberry varieties: a forensic application. Molecular Ecology, 9: $229 \sim 232$

Crandall K A, O R P Bininda-Emonds, G M Mace and R K Wayne, 2000. Considering evolutionary processes in conservation biology. Trends in Ecology and Evolution, 15: $290 \sim 295$

Crawford D J, S Brauner, M E Cosner, T F Stuessy, 1993. Use of RAPD markers to document the origin of the intergeneric hybrid Margyraceana skottsbergii (Rosaceae) on the Juan Fernandez Islands. American Journal of Botany, 80(1) : 89 $\sim 92$

Ehlers B K and H A Pedersen, 2000. Genetic variation in three species of Epipactis (Orchidaceae): geographic scale and evolutionary inferences. Biological Journal of the Linneae Society, 69: $411 \sim 430$
Falk D A and K E Holsinger, 1991. Genetics and Conservation of Rare Plants. Oxford University Press, New York

Frankham R, 1995. Conservation genetics. Annual Review of Genetics, 29: $305 \sim 327$

Gao L, S Ge and D Y Hong, 2000. Allozyme variation and population genetic structure of common wild rice, Oryza rufipogon Griff. in China. Theoretical and Applied Genetics, 101: $494 \sim 502$

Gao L Z, S Ge and D Y Hong, 2002. High levels of genetic differentiation of Oryza officinalis Wall. et Watt. from China. The Journal of Heredity (in press)

Ge S, D-Y Hong, 1994. Biosystematic studies on Adenophora potaninii complex. II . crossing experiment. Cathaya, 6: $15 \sim 26$

Ge S, D M Zhang, H Q Wang and G Y Rao, 1997. Allozyme variation in Ophiopogon xylorrhizus, an extreme endemic species of Yunnan, China. Conservation Biology, 11: 562 $\sim 565$

Ge S, D Y Hong, H Q Wang, Z Y Liu and C M Zhang, 1998. Population genetic structure and conservation of an endangered conifer, Cathaya argyrophylla (Pinaceae). International Journal of Plant Sciences, 159: $351 \sim 357$

Ge S, G C X Oliveira, B A Schaal, L Z Gao and D Y Hong, 1999a. RAPD variation within and between natural populations of the wild rice Oryza rufipogon from China and Brazil. Heredity, 82: $638 \sim 644$

Ge S, K Q Wang, D Y Hong, W H Zhang and Y G Zu, 1999b. Comparisons of genetic diversity in the endangered Adenophora lobophylla and its widespread congener, A. potanini. Conservation Biology, 13: $509 \sim 513$

Ge S, T Sang, B R Lu and D Y Hong, 2001. Rapid and reliable identification of rice genomes by RFLP analysis of PCRamplified Adh genes. Genome, 44: $1136 \sim 1142$

Gilbert J E, R V Lewis, M J Wilkinson and P D S Caligari, 1999. Developing an appropriate strategy to assess genetic variability in plant germplasm collections. Theoretical and Applied genetics, 98: $1125 \sim 1131$

Goldstein P Z, R DeSalle, G Amato and A P Vogler, 2000. Conservation genetics at the species boundary. Conservation Biology, 11: $120 \sim 131$

Hamrick J L and M J W Godt, 1990. Allozyme diversity in plant species. In: Brown A H D, M T Clegg, A L Kahler and B S Weir (eds.), Plant Population Genetics, Breeding and Genetic Resources. Sinauer Associates, Inc., Sunderland, MA, $43 \sim 63$

He T H, G Y Rao, R L You and S Ge, 1998. Mating system of Ophiopogon xylorrhizus (Liliaceae), an endangered species in southwest China. International Journal of Plant Sciences, 159: $440 \sim 445$

He T H, G Y Rao, R L You, S Ge and D M Zhang, 2001. Genetic structure and heterozygosity variation between generations of Ophiopogon xylorrhizus (Liliaceae s. l.), an endemic species in Yunnan, SW China. Biochemical Genetics, 39: $93 \sim 98$

Heywood V H and R T Watson, 1995. Global biodiversity assessment. Cambridge University Press, Cambridge

Hogbin P M and R Peakall, 1999. Evaluation of the contribution of genetic research to the management of the endangered plant Zieria prostrata. Conservation Biology, 13: $514 \sim 522$

Holsinger K E, 1996. The scope and the limits of conservation genetics. Evolution, 50: $2558 \sim 2561$ 
IUCN/SSC Orchid Specialist Group, 1996. Orchids-Status Survey and Conservation Action Plan. IUCN, Gland, Switzerland and Cambridge

Lande R, 1988. Genetics and demography in biological conservation. Science, 241: $1455 \sim 1460$

Li A, Y B Luo and S Ge, 2002. A preliminary study on conservation genetics of an endangered orchid (Paphiopedilum micranthus) from southwestern China. Biochemical Genetics, 40: (in press)

Loescheke J, J Tomiuk and S K Jain, 1994. Conservation Genetics. Birkhauser, Basel

Martin C, A Juliano, H J Newbury, B R Lu, M T Jackson and B V Ford-Lloyd, 1997. The use of RAPD markers to facilitate the identification of Oryza species within a germplasm collection. Genetic Resources and Crop Evolution, 44: 175 $\sim 183$

Meffe G K, C R Carroll, 1994. Princeples of Conservation Biology. Sinauer Associates, Inc., Sunderland, Massachusetts

Milligan B G, J Leebens-Mack and A E Strand, 1994. Conservation genetics: beyond the maintenance. Molecular Ecology, 3: $423 \sim 435$

Mitton J B and M C Grant, 1984. Associations among protein heterozygosity, growth rate, and developmental homeostasis. Annual Review of Ecology and Systematics, 15: 479 499

Morin P A, 2000. Genetic resources: opportunities and perspectives for the new century. Conservation Genetics, 1: 271 275

Moritz C, 1994. Defining evolutionary significant units for conservation. Trends in Ecology and Evolution, 9: $373 \sim 375$

Qian W, S Ge and D-Y Hong, 2001. Genetic variation within and among populations of a wild rice Oryza granulata from China detected by RAPD and ISSR markers. Theoretical and Applied Genetics, 102: $440 \sim 449$

Rio A H del, J B Bamberg and Z Huaman, 1997. Assessing changes in the genetic diversity of potato gene banks. I. Effects of seed increase. Theoretical and Applied Genetics, 95: $191 \sim 198$

Rossetto M, G Jezierski, S D Hopper and K W Dixon, 1999. Conservation genetics and clonality in two critically endangered eucalypts from the highly endemic south-western Australian flora. Biological Conservation, 88: $321 \sim 331$

Rossetto M, P K Weaver and K W Dixon, 1995. Use of RAPD analysis in devising conservation strategies for the rare and endangered Grevillea scapigera (Proteaceae). Molecular Ecology, 4: $321 \sim 329$

Ryder O A, 1986. Species conservation and systematics: the dilemma of subspecies. Trends in Ecology and Evolution, 1: $9 \sim 10$

Ryder O, A McLaren, S Brenner, Y P Zhang and K Benirschke, 2000. DNA banks for endangered animal species. Science, 288: $275 \sim 277$

Saccheri I, M Kuussaari, M Kankare, P Vikman, W Fortelius and I Hanski, 1998. Inbreeding and extinction in a butterfly metapopulation. Nature, 392: $491 \sim 494$

Savolainen O, 1994. Genetic variation and fitness: conservation lesson from pines. In: Loescheke J, J Tomiuk and S K Jain (eds), Conservation Genetics. Birkhauser, Basel, $27 \sim 36$

Savolainen O and P Hedrick, 1995. Heterozygosity and fitness: no association in Scots pine. Genetics, 140: $755 \sim 766$

Scacchi R, G D Angelis and R M Corbo, 1991. Effect of the breeding system on the genetic structure in three Cephalenthera spp. (Orchidaceae). Plant Systematic and Evolution, 176: $53 \sim 61$

Schoen D J and A H D Brown, 1993. Conservation of allelic richness in wild crop relatives is aided by assessment of genetic markers. Proceedings of the National Academy of Sciences, USA, 90: $10623 \sim 10627$

Schonewald-cox C M, S M Chambers, B MacBryde, L Thomas (eds.), 1983. Genetics and Conservation. Benjamin-Cummings Publishing, Menlo Park, CA

Smith J F, C C Burke, W L Wagner, 1996. Interspecific hybridization in natural populations of Cyrtandra (Gesneriaceae) on the Hawaiian Islands: evidence from RAPD markers. Plant Systematics and Evolution, 200: $61 \sim 77$

Soule M E and L S Hillis, 1998. No need to isolate genetics. Science, 282: $1658 \sim 1659$

Steward C N J and L Excoffier, 1996. Assessing population genetic structure and variability with RAPD data: application to Vaccinium macrocarpon (American Cranberry) . Journal of Evolutionary Biology, 9: $153 \sim 171$

Sydes M A and R Peakall, 1998. Extensive clonality in the endangered shrub Haloragodendron lucasii (Haloragaceae) revealed by allozymes and RAPDs. Molecular Ecology, 7: 87 $\sim 93$

Szalanski A L, G Steinauer, R Bischof and J Petersen, 2001. Origin and conservation genetics of the threatened Ute ladies'-tresses, Spiranthes diluvialis (Orchidaceae). American Journal of Botany, 88: $177 \sim 180$

Tanksley S D and S R McCouch, 1997. Seed banks and molecular maps: unlocking genetic potential from the wild. Science, 277: $1063 \sim 1066$

Virk P S, H J Newbury, M T Jackson and B V Ford-Lloyd, 1995. The identification of duplicate accessions within a rice germpoasm collection using RAPD analysis. Theoretical and Applied Genetics, 90: $1049 \sim 1055$

Wolfe A D, A Liston, 1998. Contributions of PCR-based methods to plant systematics and evolutionary biology. In: Soltis D E, P S Soltis and J J Doyle (eds), Molecular Systematics of Plants II: DNA Sequencing. Kluwer Academic Publishers, Norwell, MA, $43 \sim 86$

Wong K C and M Sun, 1999. Reproductive biology and conservation genetics of Goodyera procera (Orchidaceae). American Journal of Botany, 86(10): $1406 \sim 1413$

Woodruff D S, 1989. The problems of conserving genes and species. In: Weston D and M Pearl (eds., ), Conservation for the Twenty First Century, $76 \sim 88$. Oxford University Press, New York

Woodruff D S, 1990. Genetics and demography in the conservation of biodiversity. Journal of Scientific Society of Thailand, 16: $117 \sim 132$

Xie Z W, Y Q Lu, S Ge, D Y Hong and F Z Li, 2001. Clonality in wild rice, Oryza rufipogon (Poaceae) and its implications for conservation management. American Journal of Botany, 88: $1058 \sim 1064$ 